\title{
Performance optimization of visible light communication
}

\author{
Agha Yasir Ali*, Lubna Farhi, Usama Ahmed, Asad Subhan, Hussain Safdar and \\ Syeda Shaherbano Zaidi
}

Department of Electronics Engineering Sir Syed University of Engineering and Technology Karachi, Pakistan

* Corresponding Author: agyasir@ssuet.edu.pk

$\begin{array}{ll}\text { Submitted } & : 19 / 08 / 2020 \\ \text { Revised } & : 04 / 05 / 2021 \\ \text { Accepted } & : 13 / 06 / 2021\end{array}$

\begin{abstract}
This paper focuses on analyzing the data rate, bit error rate (BER), flickering, and bandwidth of visible light communication (VLC) system. The existing modulation scheme ON-OFF Keying (OOK) is modified and produces the trade-off between these parameters. The modified ON-OFF Keying (MOOK) is proposed, in which the variation in the transmitted pulses is investigated. Therefore, the square and rectangular pulses are used to transmit zero bits and one bit, respectively. The duty cycle of square pulse is increased to improve the flickering performance. Moreover, it utilized the bandwidth and leads the BER performance to deteriorate. The differential MOOK (DMOOK) modulation scheme is also proposed, in which the ON period of square pulse (zero bits) is removed. Therefore, the data rate of DMOOK is increased because the duration of zero-bit pulse is decreased. Similarly, the high bandwidth is utilized, and the BER performance deteriorates. All performance parameters are evaluated on Arduino-based hardware VLC system. We conclude that the BER performance deteriorates by improving flickering performance and data rate of the VLC system.
\end{abstract}

Keywords: Visible Light Communication; Modulation; Flickering; Data rate.

\section{INTRODUCTION}

Visible Light Communication (VLC) systems take advantage of light emitting diodes (LEDs), which can be pulsed at very high speeds without noticeable effect on the lighting output and human eye. VLC system based on the white LED has attracted much attention and has become a highly potential and valuable method for wireless communication (Liu and Zhang 2014, Lian, Vatansever et al. 2019, Rehman, Ullah et al. 2019).

Many typical modulation methods can be adopted in free space optical (FSO) communication systems, such as ON-OFF keying (OOK), pulse position modulation (PPM), digital pulse interval modulation (DPIM), and dual header pulse interval modulation (DHPIM). The OOK and PPM were firstly proposed in Kim and Jung (2015), Gao, Li et al. (2017), Ali, Zia et al. (2019), and Han, Kim et al. (2019). In this paper, the channel information is obtained without any loss of bandwidth efficiency while ensuring illumination quality by reducing the flickering effect of an OFDM based VLC system. A 6.4\% low frequency flicker is proposed in Ali, Zhang et al. (2016) and Gao, Li et al. (2017), and the light output of LED drivers varies significantly at the double-line-frequency, which not only is considered as a harmful optical flicker, but also greatly affects the effectiveness of data transmission. An OFDM-based VLC system 
with reduced flickering effects is investigated in Kim and Jung (2015), and the results show that the proposed method provides a $6.4 \%$ gain in bandwidth efficiency with a $4 \%$ reduction in flicker compared to a conventional OFDMbased method. The flicker mitigation performance of different codes is applied, and the trade-offs between flicker mitigation, code rate, and coding gain design several codes and compare their error rate and flicker mitigation performance to some codes in the VLC IEEE 802.15.7 standard (Cailean and Dimian 2017). A low flickering code is investigated in Gao, $\mathrm{Li}$ et al. (2017). The performance of code is compared on the basis of minimum Hamming distance, peak to average power ratio, run-length, and bit error rate.

The results show that a high data rate with low complexity is achieved by the proposed code in Uddin, Cha et al. (2011). Designing a low-cost VLC system is a challenge, which can provide illumination and communication link at minimum flickering and improved data rate. The aim of this paper to develop a low-cost VLC system at minimized level of flickering at sufficient brightness in the room. The transmitting and detection of high bandwidth pulses are a challenge in low-cost hardware like Arduino controller. Therefore, a novel modulation scheme is proposed to reduce flickering. The high bandwidth pulses are produced by using high speed driving circuit, and a highly efficient photo diode is used for receiving high speed pulses. The flickering problem is improved by reducing the OFF cycle of zero bits. However, the detection of high bandwidth OFF cycle pulse is a challenge; therefore, a trade-off is created between flickering, BER, bandwidth, and data rate. The BER performance is deteriorated due to increasing bandwidth at cost improved flickering. In a VLC system, nonnegative pulses are being transmitted, which makes DC biasing applicable. It maintains the minimum required brightness when no data is being transmitted.

The remainder of this paper is organized as follows. In Section II, we propose a flicker measure used to compare the codes involved in this study. In Section III, we introduce the general concepts and present two algorithms to design codes for flicker mitigation to improve coding gain. In Section IV, we compare the designed codes with the standard codes in terms of flicker mitigation and BER performance. Finally, in Section V, we conclude the paper.

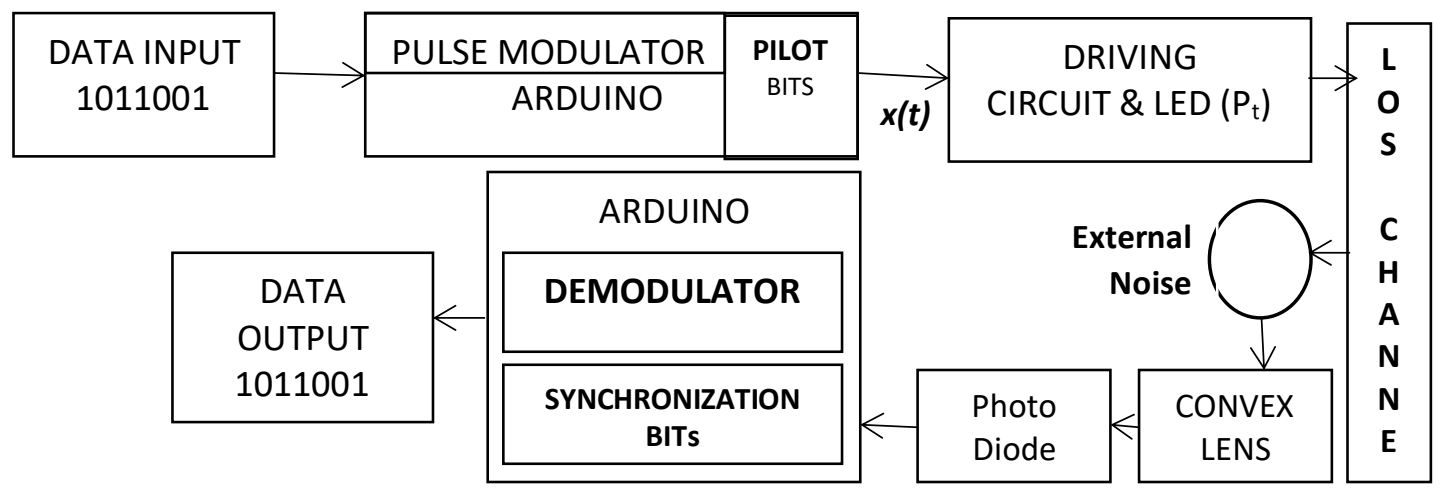

Figure 1. The proposed VLC model.

\section{THE MODEL OF PROPOSED SYSTEM}

Visible Light Communication uses intensity-modulation and direct-detection (IM/DD). Intensity modulation is obtained by varying the bias current of LEDs. A photodetector in a direct-detection receiver produces a photocurrent that is proportional to the optical power incident upon it. The modulation schemes used in VLC use power to transmit information. Let $x(t)$ and $y(t)$ denote the transmitted and received optical signals, respectively, as given in $\operatorname{Eq}(1)$. The proposed VLC system model only Line of Sight (LOS) channel is considered in $\mathrm{Eq}(4)$, and the transmitted and 
received power are shown in $\mathrm{Eq}(2)$ and $\mathrm{Eq}(3)$. The input pulse $\mathrm{x}(\mathrm{t})$ is passing through the additive white Gaussian noise (AWGN) $n(t)$ (Komine and Nakagawa 2004):

$y(t)=R h(t) * x(t)+n(t)$

$y(t)$ is the photodetector current, and $R$ represents the photo responsivity of the photodetector (in $\mathrm{A} / \mathrm{W}$ ). The time average transmitted optical power is given by

$P_{t}=\lim _{T \rightarrow \infty} \frac{1}{2 T} \int_{-T}^{T} x(t) d t$

The average received optical power generally can be determined as follows:

$P_{r}=H_{L O S}(0) P_{t(L O S)}$

where $H(0)=\int_{-\infty}^{\infty} h(t) d t$ is the channel DC gain.

$H(0)_{L O S}=\left\{\begin{array}{c}\frac{(l+1) A_{P D} \gamma \cos ^{l}(\varphi) g(\psi) T(\psi)_{\operatorname{cof}} \cos (\psi)}{2 \pi d^{2}} \\ 0, \text { else where }\end{array}\right.$

where $\mathrm{d}$ is the distance between the transmitter and receiver, $\gamma$ is the reflectance factor, 1 is the order of Lambertian emission, APD is the receiving area of photo detector, $\varphi$ is the irradiance angle, $\psi$ is the angle of incidence, and $T(\psi)_{c o f}$ is the signal transmission coefficient of an optical filter. The field of view (FOV) in $\mathrm{Eq}(5)$ is represented as $\psi c$, and $p, g(\psi)$ are refractive index and gain of the optical concentrator (Komine and Nakagawa 2004).

$g(\psi)=\left\{\begin{aligned} \frac{p^{2}}{\sin ^{2} \psi_{c}}, & 0 \leq \psi \leq \psi_{c} \\ 0, & 0 \geq \psi_{c}\end{aligned}\right.$

VLC is typically characterized by a nonnegative and noncoherent signal transmission. The block diagram of the proposed VLC transmitting and receiving systems is shown in Fig. 1. The binary input data is inserted in Arduino controller (AC) at IDE port. The AC modulates the binary data into pulses, and then pilot bits are inserted for synchronization of transmitter and receiver. The AC provides low power signal; therefore, the driving circuit is used to provide sufficient power to LED. The light is transmitted to LOS channel and received by small-scale solar panel via lens. The receiver uses AC for demodulation and synchronization. An ON-OFF keying (OOK) transmitter emits a rectangular pulse of duration $T_{\mathrm{s}}$ intensity to signify one bit and no pulse to represent zero bits. The OOK signal is $x(t)=2 P_{b} \operatorname{rect}\left(t / T_{s}\right)$ as shown in Fig 1. $T_{s}$ shows the symbol time, the shape of the $x(t)$ is of a rectangular pulse, and $P_{b}$ is bit power. The OOK modulation is modified in the proposed model, in which the digital input one bit is modulated by a high pulse, and the zero bits are subdivided into high and low $\left(T_{S}=t_{H}+t_{L}\right)$ pulses. In order to improve flickering, high pulse $t_{\mathrm{H}}$ duration keeps high and then low pulse $t_{\mathrm{L}}$ duration $t_{\mathrm{H}}>\mathrm{t}_{\mathrm{L}}$ as shown in Fig 2(b). Moreover, the flickering can be improved more by increasing $t_{H}$ and then $t_{L}\left(t_{H}>>t_{L}\right)$. The flickering is depending upon the OFF cycle $t_{L}$ of the pulse, and the OFF cycle increases the flicking in the room. However, the bit error performance decreased due to the decrease of $t_{\mathrm{L}}$. The decrease of low pulse duration $\mathrm{t}_{\mathrm{L}}$ utilizes high bandwidth; therefore, there is a trade-off between flickering, BER, and bandwidth. The BER performance is also improved by using convex lens at receiver. The differential modified OOK modulation (DMOOK) scheme is also proposed to improve the data rate. The low duration time $t_{\mathrm{H}}=0$ is removed; only $t_{\mathrm{L}}$ pulse duration exists in the zero bits of binary inputs as shown in Fig 2(c). 


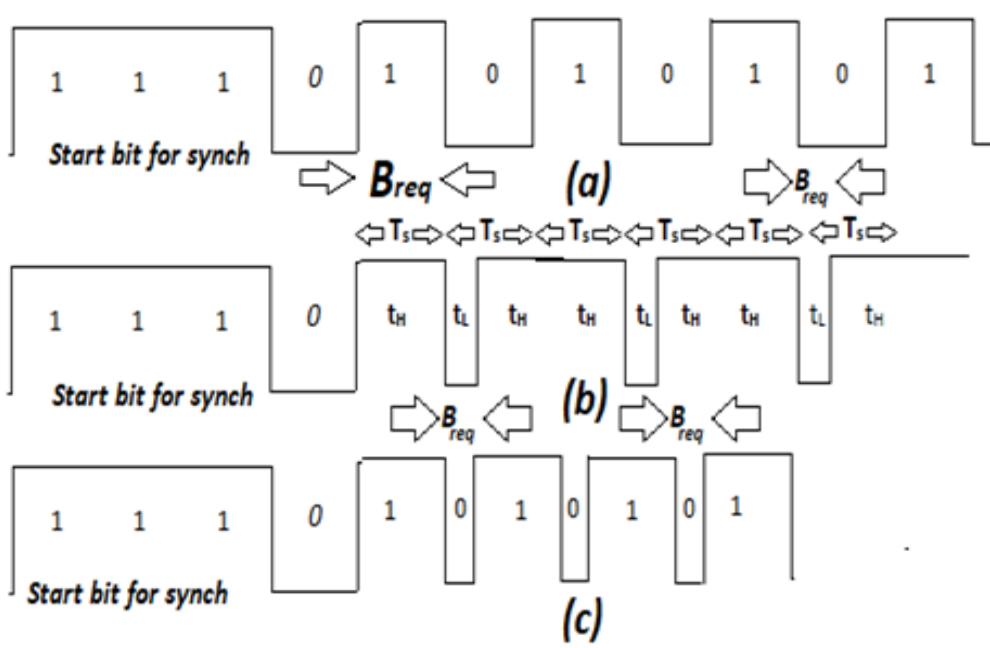

Figure 2. Signaling scheme. (a) Binary input. (b) MOOK. (c) DMOOK.

In this scheme, only the width of the low pulse is varied in accordance with the data rate $=1 / T_{s}$. The data rate is depending on $T_{s}$, and the symbol times of OOK, MOOK, and DMOOK are not the same.

\section{ANALYSIS}

The BER and flickering and bandwidth performances of the proposed MOOK and DMOOK modulation schemes are analyzed. The flickering performance increases if $t_{H}$ is greater than $t_{L}$. The MOOK has $t_{H}$ higher then $t_{L}$; therefore, MOOK has the highest flickering performance and then OOK and DMOOK. The total eight bits are transmitted in Fig. 2, which is transmitted in $8 \mathrm{~ms}$. The off duration t $\mathrm{t}_{\mathrm{L}}$ of the zero bits is measured to be $2.4 \mathrm{~ms}$ in MOOK as shown in Fig. 2(b). Similarly, the DMOOK duration of eight bits is $5.2 \mathrm{~ms}$, and OFF duration th of zero bits is measured to be $1.2 \mathrm{~ms}$. This analysis shows that MOOK has lesser flickering than DMOOK. However, DMOOK transmits eight bits in $5.2 \mathrm{~ms}$; therefore, the data rate is greater than MOOK. The Flickering performance can be obtained by

$$
\text { Flickering } \%=\frac{t_{H}-t_{L}}{t_{H}+t_{L}} \times 100
$$

The bandwidth is defined as the inverse of minimum time duration of ON or OFF pulse duration as shown in Fig. 2 (Ali, Zhang et al. 2016).

$$
B_{\text {req }}=\frac{1}{t_{L}}
$$

The bandwidths of MOOK and DMOOK are the same. However, the minimum time duration th of the zero bits is varying with bandwidth utilization. The high bandwidth pulses have low BER performances and better flickering performance.

The BER performance of MOOK and DMOOK can be derived from constellation diagram as shown in Fig. 3. As depicted in Fig. 3, the zero-bit (' $n$ ' point) location is varying between ' $\mathrm{m}$ ' to 'o' points, and the BER performance also varies. The high distance between 'o' and ' $n$ ' points obtains better BER performance, utilizes less bandwidth, 
and produces high flickering in MOOK. However, in DMOOK, $t_{\mathrm{H}}$ is zero, and only the duration of t is varying. Similarly, the higher distance between them obtains better BER performance.

The sum of error probability functions of zero bits and one bit is given as follows:

$P_{b}=\frac{1}{\sqrt{\pi \sigma}} e^{-\frac{\left(y+\sqrt{E_{n}}\right)^{2}}{\sigma^{2}}}+\frac{1}{\sqrt{\pi \sigma}} e^{-\frac{\left(y+\sqrt{E_{o}}\right)^{2}}{\sigma^{2}}}$

$P_{b}=\frac{1}{2}\left(\operatorname{erfc}\left(\sqrt{\frac{E_{n}}{\sigma}}\right)+\operatorname{erfc}\left(\sqrt{\frac{E_{o}}{\sigma}}\right)\right)$

$E_{m}<E_{n}<E_{o}$

$\mathrm{E}_{\mathrm{m}}=\mathrm{E}_{\mathrm{n}}$ condition defines OOK and $\mathrm{E}_{\mathrm{n}}<\mathrm{E}_{\mathrm{o}}$ is defined MOOK, however, in DMOOK, the

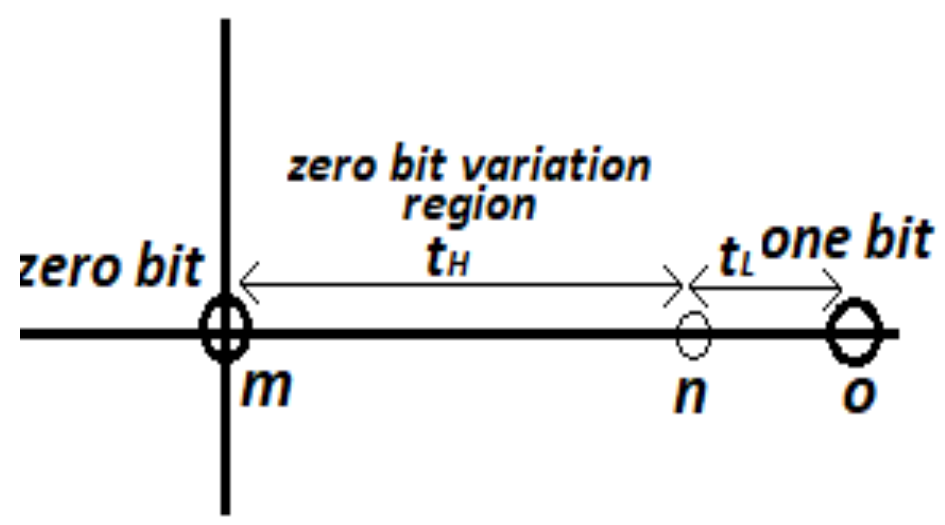

Figure 3. Constellation diagram.

error performance is the same as that of MOOK.

\section{RESULTS AND DISCUSSION}

In this section, various results regarding the performance of different parameters using OOK, MOOK, and DMOOK different modulation schemes are discussed. Fig. 4 shows the BER performance against different data rates. The results show that OOK modulation has better BER performance than MOOK and DMOOK. BER and flickering performances are inversely proportional to each other. However, in Fig. 5, the lower flickering values are considered of better performance than higher flickering values, and in BER performance, the lower values are of higher performance, and higher values are considered as low BER performance. The results in Fig. 5 show that the BER performance of OOK is better than that of MOOK and DMOOK because the constellation distance is high in the case of OOK. However, the higher constellation distance produces high flickering in the room. Therefore, the flickering 
performance of MOOK and DMOOK is better than that of OOK. The bandwidth performance of the proposed scheme and OOK scheme is obtained in Fig. 6. The results show that the OOK utilized low bandwidth but high flickering due to equal pulse duration of one and zero bits. However, the high bandwidth pulse is used in MOOK and DMOOK, but better flickering performance is obtained. The bandwidth performance is also inversely proportional to the BER performance as shown in Fig. 7. As depicted in Fig. 7, the OOK utilized low bandwidth resources and high constellation distance between one and zero bits. Therefore, BER performance was higher than that of MOOK and DMOOK.

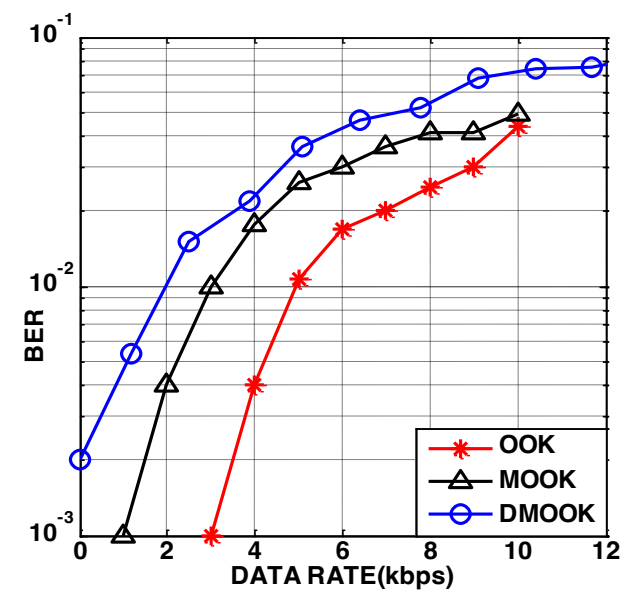

Figure 4. BER vs data rate performance of OOK, MOOK, and DMOOK.

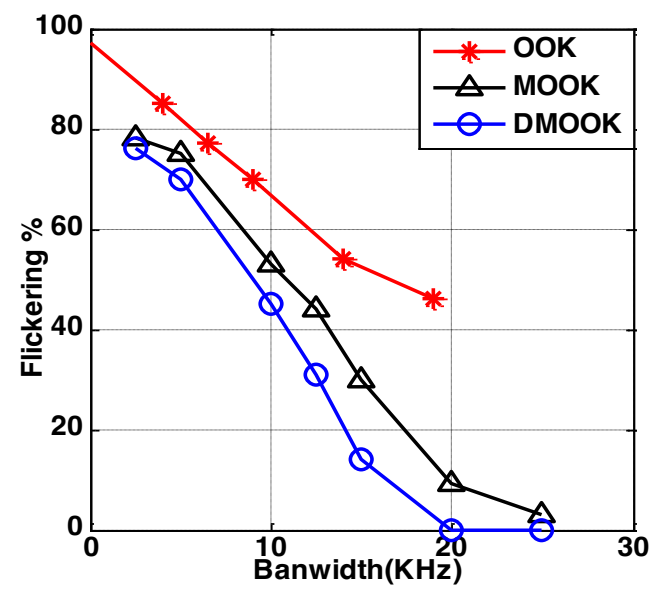

Figure 6. Flickering vs bandwidth performance of OOK, MOOK, and DMOOK.

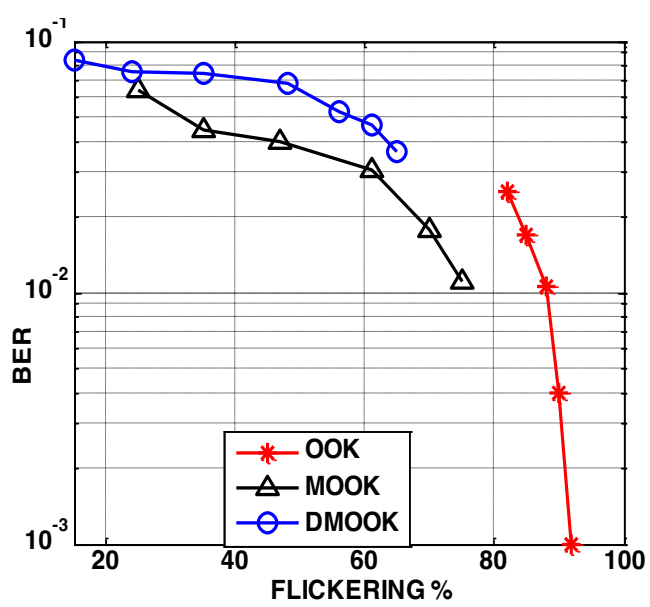

Figure 5. BER vs flickering performance of OOK, MOOK, and DMOOK.

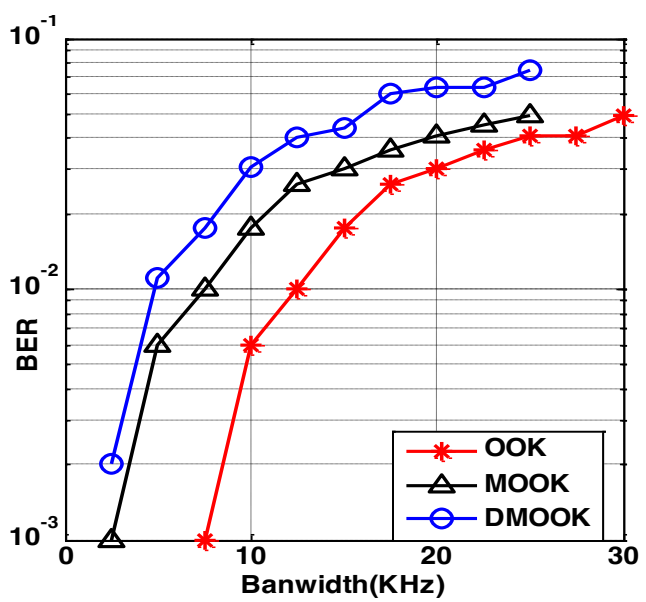

Figure 7. BER vs bandwidth performance of OOK, MOOK, and DMOOK. 


\section{CONCLUSION}

The flickering problem is mainly investigated in this study. Therefore, the proposed technique is mainly devoted to reducing the flickering in the room. The high bandwidth pulses are utilized in the proposed scheme MOOK and DMOOK to reduce the flickering significantly. The BER performance deteriorated by utilizing high bandwidth pulses. Moreover, high speed controllers are required to detect the high bandwidth pulses. Therefore, we provide a trade-off between bandwidth and BER performance. The data rate performance is improved in DMOOK as compared to OOK and MOOK because the $t_{\mathrm{L}}$ (zero bit) $<<\mathrm{t}_{\mathrm{H}}$ (one bit) pulse duration of zero bits is reduced. The BER performance is improved by using the convex lens.

\section{REFERENCES}

Ali, A. Y., Z. Zhang, A. M. Abdelgader and B. Zong (2016). "Data rate optimization in inter-cell interference environment of visible light communication." Photonic Network Communications 32(1): 133-141.

Ali, A. Y., R. Zia, L. Farhi, S. Ahmed, U. E. Laila and M. Ibrar-ul-Haque (2019). "Advanced Modulation

Scheme for Visible Light Communication." Indian Journal of Science and Technology 12: 17.

Cailean, A.-M. and M. Dimian (2017). "Impact of IEEE 802.15. 7 standard on visible light communications usage in automotive applications." IEEE Communications Magazine 55(4): 169-175.

Gao, Y., L. Li and P. K. Mok (2017). 22.8 An AC-input inductorless LED driver for visible-light-communication applications with $8 \mathrm{Mb} / \mathrm{s}$ data-rate and 6.4\% low-frequency flicker. 2017 IEEE International Solid-State Circuits Conference (ISSCC), IEEE.

Han, Y., Y. Kim and B. W. Kim (2019). "Bit-shuffle coding for flicker mitigation in visible light communication." IEEE Access 7: 150271-150279.

Kim, B. W. and S. Y. Jung (2015). "Bandwidth-Efficient Precoding Scheme with Flicker Mitigation for OFDMBased Visible Light Communications." ETRI Journal 37(4): 677-684.

Komine, T. and M. Nakagawa (2004). "Fundamental analysis for visible-light communication system using LED lights." IEEE transactions on Consumer Electronics 50(1): 100-107.

Lian, J., Z. Vatansever, M. Noshad and M. Brandt-Pearce (2019). "Indoor visible light communications, networking, and applications." Journal of Physics: Photonics 1(1): 012001.

Liu, Y. and G.-a. Zhang (2014). "A new modulation scheme of visible light communication." Optoelectronics Letters 10(4): 273-276.

Rehman, S. U., S. Ullah, P. H. J. Chong, S. Yongchareon and D. Komosny (2019). "Visible light communication: a system perspective_-overview and challenges." Sensors 19(5): 1153.

Uddin, M. S., J. S. Cha, J. Y. Kim and Y. M. Jang (2011). "Mitigation technique for receiver performance variation of multi-color channels in visible light communication." Sensors 11(6): 6131-6144. 